\title{
Radiative rates for E1, E2, M1, and M2 transitions in Ne-like Cu XX, Zn XXI and Ga XXII
}

\author{
Kanti M. Aggarwal ${ }^{a,, *}$ \\ ${ }^{a}$ Astrophysics Research Centre, School of Mathematics and Physics, Queen's University Belfast, \\ Belfast BT7 1NN, Northern Ireland, UK
}

\section{Abstract}

Energy levels, radiative rates and lifetimes are reported for the lowest 139 levels of three Ne-like ions, namely Cu XX, Zn XXI and Ga XXII. These levels mostly belong to the $2 \mathrm{~s}^{2} 2 \mathrm{p}^{6}, 2 \mathrm{~s}^{2} 2 \mathrm{p}^{5} 3 \ell, 2 \mathrm{~s} 2 \mathrm{p}^{6} 3 \ell, 2 \mathrm{~s}^{2} 2 \mathrm{p}^{5} 4 \ell, 2 \mathrm{~s} 2 \mathrm{p}^{6} 4 \ell$, and $2 \mathrm{~s}^{2} 2 \mathrm{p}^{5} 5 \ell$ configurations. For the calculations the general-purpose relativistic atomic structure package (GRASP) has been adopted. 'Comparisons are made with earlier available theoretical and experimental results, particularly among the lowest 27 levels of the $2 \mathrm{~s}^{2} 2 \mathrm{p}^{6}$ and $2 \mathrm{~s}^{2} 2 \mathrm{p}^{5} 3 \ell$ configurations. Due to paucity of similar data for higher lying levels, analogous calculations have also been performed with the flexible atomic code (FAC). These calculations help in assessing the accuracy of our calculated results, especially for the energy levels.

Received: 15 February 2018, Accepted: 9 March 2018

Keywords: Ne-like ions, energy levels, radiative rates, oscillator strengths, lifetimes

* Corresponding author.

Email address: K.Aggarwal@qub.ac.uk (Kanti M. Aggarwal) 


\section{Contents}

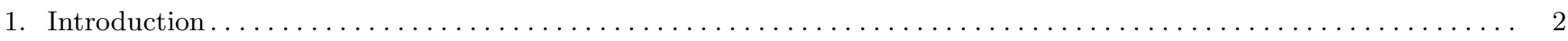

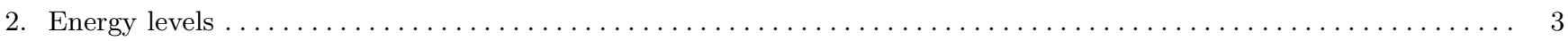

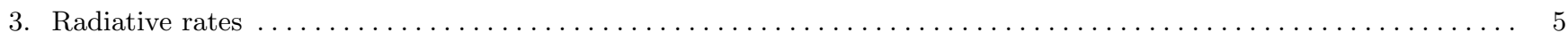

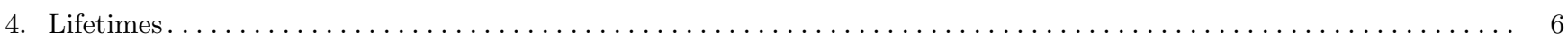

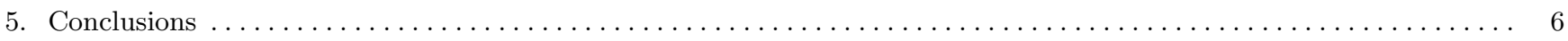

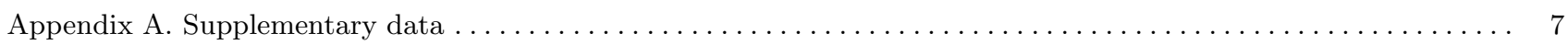

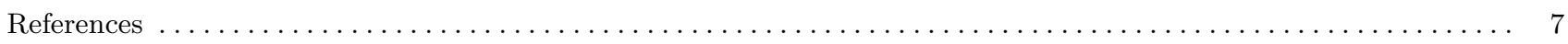

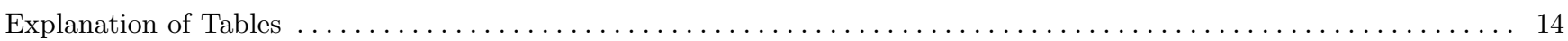

\section{Introduction}

Ne-like ions occur in a variety of plasmas, such as astrophysical, laser-produced, magnetically confined, and Z-pinch - see for example [1] and references therein. Particularly important among these are the iron group of elements (namely $\mathrm{Ti}, \mathrm{Cr}, \mathrm{Fe}$ and $\mathrm{Ni}$ ), whose lines are highly useful for the modelling and diagnostics of a range of plasmas, including astrophysical, fusion and laser generated. However, to interpret observations or to model the plasmas, information is required for several atomic parameters, such as energy levels, radiative rates and collision strengths. Therefore, over a period of time we have reported atomic data for a few Ne-like ions, namely Cl VIII [2], Fe XVII [3, 4], Ni XIX [5, 6], and very recently W LXV [7]. Similarly, many other workers have reported data for Ne-like ions. The most recent works are by Jönsson et al. [8] and Singh and Aggarwal [9], who have also provided references for earlier works.

The majority of calculations available in the literature are confined to the lowest 27 levels of the $2 \mathrm{~s}^{2} 2 \mathrm{p}^{6}$ and $2 \mathrm{~s}^{2} 2 \mathrm{p}^{5} 3 \ell$ configurations, for which those by Jönsson et al. [8] are probably the most accurate, because differences between the measured and calculated energy levels are minimal. Moreover, they have covered a wider range of ions with $12 \leq \mathrm{Z} \leq$ 36. However, very recently Singh and Aggarwal [9] (henceforth to be referred to as SA) have reported energy levels, radiative rates (A-values) and lifetimes $(\tau)$ for three Ne-like ions, namely $\mathrm{Cu}$ XX, Zn XXI and Ga XXII, among 127 levels of the $2 \mathrm{~s}^{2} 2 \mathrm{p}^{6}, 2 \mathrm{~s}^{2} 2 \mathrm{p}^{5} 3 \ell, 2 \mathrm{~s} 2 \mathrm{p}^{6} 3 \ell, 2 \mathrm{~s}^{2} 2 \mathrm{p}^{5} 4 \ell, 2 \mathrm{~s} 2 \mathrm{p}^{6} 4 \ell$, and $2 \mathrm{~s}^{2} 2 \mathrm{p}^{5} 5 \ell(\ell \leq 3)$ configurations. We are not aware of any astrophysical importance of these ions, but they are useful for lasing and fusion plasmas [10-12]. For the calculations, they have adopted two independent atomic structure codes, namely the general-purpose relativistic atomic structure package (GRASP) and the flexible atomic code (FAC) of Gu [13]. Both of these codes are freely available on the websites http://amdpp.phys.strath.ac.uk/UK_APAP/codes.html and https://www-amdis.iaea.org/FAC/, respectively. It was mainly done for the assessment of accuracy, particularly for the energy levels, because prior similar data, experimental or theoretical, for most of the levels for these three ions do not exist. Therefore, based on the two calculations they concluded that both codes provide 'comparable energies'. However, we notice that for many levels of all three ions the differences between the two sets of energies are significant, i.e. up to 1.5 Ryd - see for example, levels 111-127 of $\mathrm{Cu}$ XX, 112-119 of Zn XXI and 111-116 of Ga XXII in their tables 1-3. In our long experience for a wide 
range of ions we have not noticed such large differences between calculations with these two codes, particularly when the same level of CI (configuration interaction) has been used. Additionally, in the absence of any other similar data, it is difficult to know which set of energies is more accurate. Apart from this, there are other reasons to perform yet another calculation, as discussed below.

The 127 levels considered by SA [9] are not the lowest in energy, because some from $2 \mathrm{~s}^{2} 2 \mathrm{p}^{5} 5 \mathrm{~g}$ intermix. They have included limited CI, among 51 configurations, namely $2 \mathrm{~s}^{2} 2 \mathrm{p}^{6}, 2 \mathrm{~s}^{2} 2 \mathrm{p}^{5} n \ell(3 \leq n \leq 7$, but $\ell \leq 3), 2 \mathrm{~s} 2 \mathrm{p}^{6} n \ell(3 \leq n \leq$ 7 , but $\ell \leq 3), 2 \mathrm{~s}^{2} 2 \mathrm{p}^{4} 3 \ell 3 \ell^{\prime}$, and $2 \mathrm{~s}^{2} 2 \mathrm{p}^{4} 3 \ell 3 \ell^{\prime}$. These configurations generate 1016 levels in total, but there is scope for improvement by including additional CI, particularly from the neglected configurations with $\ell>3$. More importantly, they have reported A-values only for the resonance transitions, i.e. from the ground level alone, whereas for accurate modelling of plasmas data are required for all transitions. Finally, for the reliability and accuracy of atomic data, which can be confidently applied, multiple calculations are preferred as emphasised by [14, 15].

\section{Energy levels}

In our calculations we adopt the same GRASP0 version as by SA [9]. It was originally developed by Grant et al. [16] but has been considerably improved and updated by one of the authors, i.e. P.H. Norrington. For the optimisation of the orbitals we use the option of 'extended average level' (EAL), in which a weighted (proportional to $2 j+1$ ) trace of the Hamiltonian matrix is minimised. The contributions of higher relativistic operators, namely Breit and quantum electrodynamic effects (QED), are also included. Although these effects are more important for the heavy ions, their contributions improve the accuracy of calculated results as may be noted from tables 1-3 of [9]. For the calculations, we have gradually increased the CI and our final ones have been performed with 64 configurations, namely $2 \mathrm{~s}^{2} 2 \mathrm{p}^{6}, 2 \mathrm{~s}^{2} 2 \mathrm{p}^{5} 3 \ell$, $2 \mathrm{~s} 2 \mathrm{p}^{6} 3 \ell, 2 \mathrm{~s}^{2} 2 \mathrm{p}^{5} 4 \ell, 2 \mathrm{~s} 2 \mathrm{p}^{6} 4 \ell, 2 \mathrm{~s}^{2} 2 \mathrm{p}^{5} 5 \ell, 2 \mathrm{~s} 2 \mathrm{p}^{6} 5 \ell, 2 \mathrm{~s}^{2} 2 \mathrm{p}^{5} 6 \mathrm{~s} / \mathrm{p} / \mathrm{d}, 2 \mathrm{~s}^{2} 2 \mathrm{p}^{5} 7 \mathrm{~s} / \mathrm{p} / \mathrm{d},\left(2 \mathrm{~s}^{2} 2 \mathrm{p}^{4}\right) 3 \mathrm{~s} 3 \mathrm{p}, 3 \mathrm{~s} 3 \mathrm{~d}, 3 \mathrm{p} 3 \mathrm{~d}, 3 \mathrm{~s}^{2}, 3 \mathrm{p}^{2}, 3 \mathrm{~d}^{2}$, $3 \mathrm{~s} 4 \ell, 3 \mathrm{~s} 5 \ell, 3 \mathrm{p} 4 \ell, 3 \mathrm{p} 5 \ell, 3 \mathrm{p} 5 \ell, 3 \mathrm{~d} 4 \ell$, and $3 \mathrm{~d} 5 \ell$, which generate 3948 levels (or configuration state functions, CSF) in total. Inclusion of further CI has not been possible in the version of the GRASP code adopted here, but is definitely possible though other versions, particularly GRASP2K [17], as has been done by Jönsson et al. [8]. However, we will assess the effect of additional CI by other means, as discussed below.

For the assessment of accuracy of energy levels and to gauge the significance (if any) of additional CI, we have performed analogous calculations with FAC. This is also a fully relativistic code and the past experience (on a wide range of ions) shows that the calculated energy levels are generally in (close) agreement with those with GRASP or any other atomic structure code. Apart from this it has an added advantage of efficiency and the possibility of including a very (very) large CI. Therefore, we have performed three calculations with increasing CI, which are: (i) FAC1, which includes 1112 levels of (almost) the same configurations as included by SA [9], i.e. $2^{*} 8,\left(2^{*} 7\right) 3^{*} 1,4^{*} 1,5^{*} 1,6^{*} 1,7^{*} 1$, $\left(2 \mathrm{~s}^{2} \mathrm{p}^{5}\right) 3^{*} 2$, and $\left(2 \mathrm{~s}^{2} 2 \mathrm{p}^{4}\right) 3^{*} 2$, (ii) $\mathrm{FAC} 2$, which includes 17729 levels arising from all possible combinations of $2^{*} 8$, $\left(2^{*} 7\right) 3^{*} 1,4^{*} 1,5^{*} 1,6^{*} 1,7^{*} 1,\left(2^{*} 6\right) 3^{*} 2,3^{*} 14^{*} 1,3^{*} 15^{*} 1,3^{*} 16^{*} 1$, and $3^{*} 17^{*} 1$, and finally (iii) FAC3, which includes a total of 93437 levels, the additional ones arising from $\left(2^{*} 6\right) 4^{*} 15^{*} 1,4^{*} 16^{*} 1,4^{*} 17^{*} 1,5^{*} 16^{*} 1,5^{*} 17^{*} 1,6^{*} 17^{*} 1$, and $2 * 53 * 3$. As an example, the energy spans of the levels in these calculations for Zn XXI are 190, 240 and 290 Ryd, respectively, whereas in GRASP is up to 212 Ryd.

Since, as stated above, most of the calculations (and measurements) are confined to the lowest 27 levels of the $2 \mathrm{~s}^{2} 2 \mathrm{p}^{6}$ and $2 \mathrm{~s}^{2} 2 \mathrm{p}^{5} 3 \ell$ configurations, we make detailed comparisons for these in Tables A, B and C for Cu XX, Zn XXI and 
Ga XXII, respectively. Included in these tables are the experimental energies, compiled by the NIST ((National Institute of Standards and Technology)) team from various sources, and available at the website

http://www.nist.gov/pml/data/asd.cfm, earlier calculations of Jönsson et al. [8] and SA [9], and our work with GRASP and FAC. Measurements are available for most levels of $\mathrm{Cu}$ XX [18], but for only a few for other ions. It is clear from these tables that the calculated energies of SA with the GRASP and FAC codes do not match for any ion under consideration, and differ by up to $\sim 0.08$ Ryd. These differences are not very significant and are often observed between any two calculations, but what is important is that their energies with FAC differ more (by up to $\sim 0.2$ Ryd) with our calculations, i.e. their results cannot be reproduced. It may be worth noting here that their calculations with both the GRASP and FAC codes had similar discrepancies in the past, for a range of ions, see for example the energy levels of five Br-like ions [19] with $38 \leq \mathrm{Z} \leq 42$ and F-like W LXVI [20, 21].

Energies obtained by SA [9] with the GRASP code are comparable with our similar calculations with the same CI, but not listed in Tables A, B and C. However, the effect of additional CI included in our present calculations with GRASP is up to $\sim 0.1$ Ryd, on the lowest 27 levels of Ne-like ions. Almost for all levels of the three ions, our energies are consistently higher than those of SA, and hence agree more closely with those of NIST and Jönsson et al. [8]. However, differences between the NIST and theoretical energies of Jönsson et al. are insignificant, but are up to $\sim 0.08$ Ryd with our calculations with GRASP, and the latter are invariably lower. This may be partly due to the corresponding differences in the calculations of ground level energies and partly due to the larger CI included by Jönsson et al. On the other hand, our energies obtained with FAC are invariably lower than all other theoretical and experimental results, and an inclusion of large(r) CI has an insignificant effect (of less than 0.05 Ryd), and the discrepancies with those of NIST are up to 0.25 Ryd. Therefore, for the levels and the ions discussed here an inclusion of larger CI in the FAC calculations has been of no advantage, and what has been included in the GRASP appears to be sufficient for the accurate and reliable determination of energy levels.

A notable exception in Tables A, B and C is the $2 \mathrm{~s}^{2} 2 \mathrm{p}^{5} 3 \mathrm{~d}{ }^{1} \mathrm{P}_{1}^{o}$ level of $\mathrm{Zn}$ XXI for which all theoretical energies are lower than of NIST, by up to 0.45 Ryd. We have performed several other calculations with differing amount of CI, with both GRASP and FAC, but are unable to get an energy closer to the NIST value. Therefore, Jönsson et al. [8] speculated a possibility of error in the determination of this measured result and suggested re-measurement. However, a closer examination of the original papers on which the NIST compilations are based reveals that for the concerned level, the measurement has been performed first by Burkhalter et al. [22] and later by Boiko et al. [23] from laser produced plasma sources, and they both reported $\lambda$ for the $3 \mathrm{C}\left(2 \mathrm{~s}^{2} 2 \mathrm{p}^{6}{ }^{1} \mathrm{~S}_{0}-2 \mathrm{~s}^{2} 2 \mathrm{p}^{5} 3 \mathrm{~d}{ }^{1} \mathrm{P}_{1}^{o}\right)$ transition to be $10.462 \AA$ with an accuracy of $\pm 2 \mathrm{~m} \AA$, or equivalently an energy of $87.103( \pm 0.016)$ Ryd, which agrees excellently with our result with GRASP or that of Jönsson et al. with GRASP2K. Additionally, based on the Cowan's code [24] they calculated $\lambda$ to be 10.47 and $10.459 \AA$ A, i.e. 87.036 and 87.128 Ryd, respectively. This further confirms that there is no discrepancy between theory and measurement for this level. This anomaly has also been confirmed by one of the NIST team member (Alexander Kramida) and will (hopefully) be corrected soon on their website.

For higher lying levels the only results available in the literature with which to compare are those by SA [9] for all three ions, and by Abdelaziz et al. [25] for $\mathrm{Cu}$ XX who have adopted the Cowan's code [24] based on Hartree-Fock-Slatter method. They have included 65 levels of the $2 \mathrm{~s}^{2} 2 \mathrm{p}^{6}, 2 \mathrm{~s}^{2} 2 \mathrm{p}^{5} 3 \ell$ and $2 \mathrm{~s}^{2} 2 \mathrm{p}^{5} 4 \ell$ configurations, but have neglected those from $2 \mathrm{~s} 2 \mathrm{p}^{6} 3 \ell$, which intermix. Additionally, several of their levels are non-degenerate in energy - see for example, $28 / 29$, 
41/42/43, 52/53, and 63/64 in their table 4. Therefore, we will not make comparisons with their results. Similarly, the results obtained by SA with FAC are not correct, as already stated, and those obtained with GRASP are comparable with our similar calculations with limited CI. Since the energies obtained in our present calculations with a larger CI are more accurate, as partly demonstrated in Tables A, B and C, we list these in Tables 1-3 for all three ions. Also included in these tables, for comparison purposes, are the results with our largest calculations with FAC. The $2 \mathrm{~s}^{2} 2 \mathrm{p}^{6}, 2 \mathrm{~s}^{2} 2 \mathrm{p}^{5} 3 \ell$, $2 \mathrm{~s} 2 \mathrm{p}^{6} 3 \ell, 2 \mathrm{~s}^{2} 2 \mathrm{p}^{5} 4 \ell, 2 \mathrm{~s} 2 \mathrm{p}^{6} 4 \ell, 2 \mathrm{~s}^{2} 2 \mathrm{p}^{5} 5 \ell$, and $2 \mathrm{~s} 2 \mathrm{p}^{6} 5 \ell(25)$ configurations generate 157 levels in total, but their energies are not the lowest, because some from other configurations intermix, such as of $2 \mathrm{~s}^{2} 2 \mathrm{p}^{5} 6 \ell$. Therefore, we are restricting our results to the lowest 139 for which levels of other configurations do not mix.

As is often the case for most calculations, the $\mathrm{LSJ}^{\pi}$ designations provided in Tables $1-3$ are not unique for all levels, because for a few the eigenvector from a particular level/configuration dominates for more than one. This has been discussed several times in the past, by many workers. Therefore, to help comparisons with other calculations sometimes mixing coefficients are listed, as has been done by Jönsson et al. [8] and SA [9]. Since we too have similar compositions, we are not listing these in Tables 1-3, but the results can be provided by the author on request. Additionally, the level orderings from GRASP and FAC are (generally) similar with only a few exceptions, but it is not always possible to find a perfect correspondence between the two calculations, because of different levels of CI included and the designations (nomenclatures) provided by the two codes. Nevertheless, in magnitude both calculations agree within about 0.2 Ryd with the FAC energies being lower, as was the case for the lowest 27 levels in Tables A, B and C. In spite of using a larger CI in the FAC calculations our results with GRASP are expected to be (comparatively) more accurate, and this conclusion is based on the detailed comparisons discussed earlier for the lowest 27 levels, for all three ions.

\section{Radiative rates}

In Tables 4-6 we list our calculated results with the GRASP code for transition energies (wavelengths, $\lambda_{j i}$ in $\AA$ ), radiative rates (A-values, in $\mathrm{s}^{-1}$ ), oscillator strengths (f-values, dimensionless), and line strengths (S-values, in atomic units, 1 a.u. $=6.460 \times 10^{-36} \mathrm{~cm}^{2} \mathrm{esu}^{2}$ ) for electric dipole (E1) transitions in Ne-like ions Cu XX, Zn XXI and Ga XXII. These results are listed among the same 139 levels given in Tables 1-3. Although results have been obtained in both the velocity (Coulomb) and length (Babushkin) gauges, those from the latter are listed alone, because these are (generally) considered to be more accurate. However, their ratio $(\mathrm{R})$ is included in these tables. For a majority of strong transitions (with $\mathrm{f} \geq 0.01) \mathrm{R}$ is close $(\mathrm{r}$ ) to unity but deviations may be large for a few (very) weak ones. For (more) accurate modelling of plasmas and determination of lifetimes (to be discussed later) not only data for all E1 transitions are required, but also for other types, namely magnetic dipole (M1), electric quadrupole (E2) and magnetic quadrupole (M2). Therefore, included in these tables are our A-values for these transitions also and the corresponding data for f- or S-values can be obtained using Eqs. (1-5) given in [4]. However, for brevity only transitions from the lowest 5 to higher excited levels are listed in Tables 4-6, but full tables in the ASCII format are available online in the electronic version.

In Table D we compare our f-values with those of Jönsson et al. [8] for all E1 transitions, but from the lowest 5 levels. This will give an idea about the accuracy of the results obtained. For most transitions, particularly with $\mathrm{f} \geq 0.01$, the two sets of calculations agree within $10 \%$. This is highly satisfactory and encouraging, and confirms, once more, that small differences in energy levels do not adversely affect the calculations of A-values. However, differences for a few (very) weak transitions, such as $3-13$ and $5-7$, are up to $25 \%$. It is very difficult to assess with confidence the accuracy 
of such weak transitions ( $\mathrm{f} \leq 10^{-4}$ ) because some variations in mixing coefficients can often make large differences due to additive or cancellation effects of their small magnitudes. Jönsson et al. have also reported A-values for M1, E2 and M2 transitions, for which there are no major discrepancies with our results. However, for a few transitions some discrepancies are unavoidable, and these are reflected in the determination of $\tau$, discussed below.

\section{Lifetimes}

The lifetime $\tau$ of a level $j$ can be determined from the A-values as it is equal to $1.0 / \Sigma_{i} \mathrm{~A}_{j i}$, where the summation runs over all types of transitions, i.e. E1, E2, M1, and M2. It is a measurable quantity but to the best of our knowledge no experiments have yet been performed for transitions/levels of the ions under consideration. However, Jönsson et al. [8] and SA [9] have listed $\tau$ and in Table E we make comparisons with their results for the lowest 27 levels of $\mathrm{Cu}$ XX, Zn XXI and Ga XXII. As expected there are no significant discrepancies among the three sets of results, and the only level which stands out is $2 \mathrm{p}^{5} 3 \mathrm{~s}^{3} \mathrm{P}_{2}^{o}$ of $\mathrm{Cu} \mathrm{XX}$ for which our value is lower by a factor of two. The only transition contributing to this level is 1-2 M2 for which our A-value is $1.27 \times 10^{6} \mathrm{~s}^{-1}$, whereas of Jönsson et al. and SA are $6.30 \times 10^{5} \mathrm{~s}^{-1}$, and $6.81 \times 10^{5} \mathrm{~s}^{-1}$, respectively, and hence the difference. Since this is a very weak transition with $\mathrm{f}=1.12 \times 10^{-7}$, it cannot be said with confidence which result is more accurate. For future comparisons, with experimental or theoretical data, our calculated values of $\tau$ are included in Tables 1-3, and based on the comparisons discussed here and earlier for the A-values, our results are expected to be accurate $\sim 20 \%$, for most levels/transitions.

\section{Conclusions}

In this paper, energies for the lowest 139 levels among the $2 \mathrm{~s}^{2} 2 \mathrm{p}^{6}, 2 \mathrm{~s}^{2} 2 \mathrm{p}^{5} 3 \ell, 2 \mathrm{~s} 2 \mathrm{p}^{6} 3 \ell, 2 \mathrm{~s}^{2} 2 \mathrm{p}^{5} 4 \ell, 2 \mathrm{~s} 2 \mathrm{p}^{6} 4 \ell, 2 \mathrm{~s}^{2} 2 \mathrm{p}^{5} 5 \ell$, and $2 \mathrm{~s} 2 \mathrm{p}^{6} 5 \ell$ configurations are reported for three Ne-like ions, $\mathrm{Cu}$ XX, Zn XXI and Ga XXII. The GRASP code has been adopted for the calculations and a reasonably large CI among 64 configurations (3948 CSF) has been included, which has been found to be sufficient, based on comparisons with other available theoretical and experimental energy levels. Nevertheless, calculations have also been performed with much larger CI (including 93437 CSF) with FAC to assess the effect and the accuracy. However, inclusion of such a large CI is not found to be beneficial for the levels considered in the paper. Based on multiple comparisons, particularly among the lowest 27 levels, our energies are assessed to be accurate to within $0.1 \mathrm{Ryd}$, for all ions.

Further calculations for A-values (and other related parameters) have also been performed for four types of transitions, namely E1, E2, M1, and M2. These results are also listed, for all transitions among the 139 levels, and hence are significantly more extensive than currently available in the literature. Comparisons with the existing literature have been possible, mostly among the lowest 27 levels, for which no major discrepancies are found. Based on the comparisons made with other calculations, for both A-values and $\tau$, our results are assessed to be accurate to $\sim 20 \%$. However, this assessment of accuracy applies mostly to the strong transitions with large f-values. For weak(er) transitions the reported A-values may be less certain. 


\section{Acknowledgments}

We thank the anonymous referee whose simple observation prompted us to resolve the discrepancy between theory and measurement for one of the levels of Zn XXI and Alexander Kramida who very graciously and promptly confirmed the error in the NIST listing.

\section{Appendix A. Supplementary data}

Owing to space limitations, only parts of Tables 4-6 are presented here, but full tables are being made available as supplemental material in conjunction with the electronic publication of this work. Supplementary data associated with this article can be found, in the online version, at doi:nn.nnnn/j.adt.2018.nn.nnn.

\section{References}

\section{References}

[1] P.L. Hagelstein, R.K. Jung, At. Data Nucl. Data Tables 37 (1987) 121.

[2] M. Mohan, K.M. Aggarwal, Phys. Scr. 49 (1994) 62.

[3] K.M. Aggarwal, F.P. Keenan, A.Z. Msezane, Astrophys. J. Suppl. 144 (2003) 169.

[4] K.M. Aggarwal, F.P. Keenan, R. Kisielius, Astron. Astrophys. 420 (2004) 783.

[5] K.M. Aggarwal, F.P. Keenan, Astron. Astrophys. 460 (2006) 959.

[6] K.M. Aggarwal, F.P. Keenan, Astron. Astrophys. 488 (2008) 365.

[7] K.M. Aggarwal, F.P. Keenan, At. Data Nucl. Data Tables 111-112 (2016) 187.

[8] P. Jönsson et al., At. Data Nucl. Data Tables 100 (2014) 1.

[9] N. Singh and S. Aggarwal, Pramana - J. Phys. 89 (2017) 79.

[10] R.C. Elton, T.N. Lee, W.A. Molander, J. Opt. Soc. Am. B 4 (1987) 539.

[11] M. Sm̌íd, O. Renner, F.B. Rosmej, D. Khaghani, Phys. Scr. T 161 (2014) 014020.

[12] O. Renner, M. Š̌íd, D. Khaghani, F.B. Rosmej, J. Phys. Conf. Series 688 (2016) 012091.

[13] M.F. Gu, Can. J. Phys. 86 (2008) 675.

[14] H-K. Chung et al., J. Phys. D 49 (2016) 363002.

[15] K.M. Aggarwal, Atoms 5 (2017) 5040037.

[16] I.P. Grant, B.J. McKenzie, P.H. Norrington, D.F. Mayers, N.C. Pyper, Comput. Phys. Commun. 21 (1980) 207.

[17] P. Jönsson, X. He, C.F. Fischer, I.P. Grant, Comput. Phys. Commun. 177 (2007) 597.

[18] J. Sugar, A. Musgrove, J. Phys. Chem. Ref. Data 19 (1990) 527.

[19] K.M. Aggarwal, F.P. Keenan, Phys. Scr. 89 (2014) 125404.

[20] K.M. Aggarwal, Chin. Phys. B 25 (2016) 043201. 
[21] K.M. Aggarwal, Atoms 4 (2016) 4030024.

[22] P.G. Burkhalter, D.J. Nagel, R.D. Cowan, Phys. Rev. A 11 (1975) 782.

[23] V.A. Boiko, A.Ya. Faenov, S.A. Pikuz, J. Quant. Spect. Rad. Transf. 19 (1978) 11.

[24] R.D. Cowan, The theory of atomic structure and spectra, Univ. of Calif. Press (1981).

[25] W.S. Abdelaziz, M.A. Khedr, M.E. Ahmed, L. Gaabour, T. El-Shirbini, Opt. Phot. J. 4 (2014) 54. 
Table A

Comparison of energies (in Ryd) for the lowest 27 levels of $\mathrm{Cu}$ XX.

\begin{tabular}{|c|c|c|c|c|c|c|c|c|c|c|}
\hline Index & Configuration & Level & NIST & GRASP2K & GRASP1 & GRASP2 & FAC1a & FAC1b & FAC2 & FAC3 \\
\hline 1 & $2 s^{2} 2 p^{6}$ & ${ }^{1} \mathrm{~S}_{0}$ & 0.0000 & 0.0000 & 0.0000 & 0.0000 & 0.0000 & 0.0000 & 0.0000 & 0.0000 \\
\hline 2 & $2 \mathrm{~s}^{2} 2 \mathrm{p}^{5} 3 \mathrm{~s}$ & ${ }^{3} \mathrm{P}_{2}^{o}$ & 70.8717 & 70.8537 & 70.6919 & 70.7742 & 70.7705 & 70.6375 & 70.6174 & 70.6035 \\
\hline 3 & $2 s^{2} 2 p^{5} 3 s$ & ${ }^{1} \mathrm{P}_{1}^{2}$ & 71.0392 & 71.0219 & 70.8653 & 70.9440 & 70.9535 & 70.8031 & 70.7827 & 70.7694 \\
\hline 4 & $2 s^{2} 2 p^{5} 3 s$ & ${ }^{3} \mathrm{P}_{0}^{o}$ & 72.3906 & 72.3830 & 72.2166 & 72.3024 & 72.2977 & 72.1562 & 72.1332 & 72.1195 \\
\hline 5 & $2 s^{2} 2 p^{5} 3 s$ & ${ }^{3} \mathrm{P}_{1}^{0}$ & 72.4917 & 72.4797 & 72.3172 & 72.4005 & 72.4053 & 72.2515 & 72.2283 & 72.2150 \\
\hline 6 & $2 s^{2} 2 p^{5} 3 p$ & ${ }^{3} \mathrm{~S}_{1}$ & 73.5454 & 73.5319 & 73.3742 & 73.4594 & 73.4435 & 73.3253 & 73.3125 & 73.2979 \\
\hline 7 & $2 s^{2} 2 p^{5} 3 p$ & ${ }^{3} \mathrm{D}_{2}$ & 73.7969 & 73.7833 & 73.6392 & 73.7167 & 73.7184 & 73.5810 & 73.5642 & 73.5510 \\
\hline 8 & $2 s^{2} 2 p^{5} 3 p$ & ${ }^{3} \mathrm{D}_{3}$ & 74.0458 & 74.0317 & 73.8841 & 73.9649 & 73.9592 & 73.8301 & 73.8134 & 73.7998 \\
\hline 9 & $2 s^{2} 2 p^{5} 3 p$ & ${ }^{1} \mathrm{P}_{1}$ & 74.1171 & 74.1027 & 73.9583 & 74.0400 & 74.0387 & 73.9021 & 73.8843 & 73.8710 \\
\hline 10 & $2 s^{2} 2 p^{5} 3 p$ & ${ }^{3} \mathrm{P}_{2}$ & 74.3009 & 74.2868 & 74.1445 & 74.2200 & 74.2258 & 74.0841 & 74.0666 & 74.0535 \\
\hline 11 & $2 s^{2} 2 p^{5} 3 p$ & ${ }^{3} \mathrm{P}_{0}$ & & 74.8788 & 74.7588 & 74.8281 & 74.8408 & 74.6842 & 74.6676 & 74.6526 \\
\hline 12 & $2 s^{2} 2 p^{5} 3 p$ & ${ }^{3} \mathrm{D}_{1}$ & 75.2641 & 75.2555 & 75.1061 & 75.1907 & 75.1872 & 75.0452 & 75.0252 & 75.0119 \\
\hline 13 & $2 s^{2} 2 p^{5} 3 p$ & $\begin{array}{l}{ }^{3} \mathrm{P}_{1} \\
\end{array}$ & 75.6456 & 75.6365 & 75.4856 & 75.5685 & 75.5648 & 75.4238 & 75.4043 & 75.3909 \\
\hline 14 & $2 s^{2} 2 p^{5} 3 p$ & ${ }^{1} \mathrm{D}_{2}$ & 75.6925 & 75.6833 & 75.5343 & 75.6157 & 75.6162 & 75.4697 & 75.4499 & 75.4368 \\
\hline 15 & $2 s^{2} 2 p^{5} 3 p$ & ${ }^{1} \mathrm{~S}_{0}$ & 76.6090 & 76.6074 & 76.6747 & 76.6808 & 76.7670 & 76.4945 & 76.4922 & 76.4620 \\
\hline 16 & $2 \mathrm{~s}^{2} 2 \mathrm{p}^{5} 3 \mathrm{~d}$ & ${ }^{3} \mathrm{P}_{0}^{o}$ & 77.5448 & 77.5365 & 77.3901 & 77.4729 & 77.4471 & 77.3028 & 77.2885 & 77.2737 \\
\hline 17 & $2 s^{2} 2 p^{5} 3 d$ & ${ }^{3} \mathrm{P}_{1}^{o}$ & 77.6474 & 77.6328 & 77.4886 & 77.5713 & 77.5468 & 77.4011 & 77.3862 & 77.3714 \\
\hline 18 & $2 \mathrm{~s}^{2} 2 \mathrm{p}^{5} 3 \mathrm{~d}$ & ${ }^{3} \mathrm{~F}_{4}^{o}$ & 77.8230 & 77.8061 & 77.6721 & 77.7473 & 77.7263 & 77.5843 & 77.5682 & 77.5534 \\
\hline 19 & $2 s^{2} 2 p^{5} 3 d$ & ${ }^{3} \mathrm{P}_{2}^{0}$ & 77.8290 & 77.8140 & 77.6720 & 77.7551 & 77.7326 & 77.5846 & 77.5692 & 77.5538 \\
\hline 20 & $2 \mathrm{~s}^{2} 2 \mathrm{p}^{5} 3 \mathrm{~d}$ & ${ }^{3} \mathrm{~F}_{3}^{o}$ & 77.8681 & 77.8538 & 77.7222 & 77.8001 & 77.7778 & 77.6289 & 77.6116 & 77.5964 \\
\hline 21 & $2 \mathrm{~s}^{2} 2 \mathrm{p}^{5} 3 \mathrm{~d}$ & ${ }^{1} \mathrm{D}_{2}^{o}$ & 78.0302 & 78.0134 & 77.8832 & 77.9637 & 77.9411 & 77.7897 & 77.7704 & 77.7554 \\
\hline 22 & $2 \mathrm{~s}^{2} 2 \mathrm{p}^{5} 3 \mathrm{~d}$ & ${ }^{3} \mathrm{D}_{3}^{o}$ & 78.1367 & 78.1229 & 77.9900 & 78.0719 & 78.0527 & 77.8962 & 77.8772 & 77.8625 \\
\hline 23 & $2 \mathrm{~s}^{2} 2 \mathrm{p}^{5} 3 \mathrm{~d}$ & ${ }^{3} \mathrm{D}_{1}^{o}$ & 78.6105 & 78.5948 & 78.4870 & 78.5594 & 78.5407 & 78.3764 & 78.3560 & 78.3387 \\
\hline 24 & $2 \mathrm{~s}^{2} 2 \mathrm{p}^{5} 3 \mathrm{~d}$ & ${ }^{3} \mathrm{~F}_{2}^{o}$ & 79.3815 & 79.3787 & 79.2339 & 79.3150 & 79.2971 & 79.1420 & 79.1217 & 79.1066 \\
\hline 25 & $2 \mathrm{~s}^{2} 2 \mathrm{p}^{5} 3 \mathrm{~d}$ & ${ }^{3} \mathrm{D}_{2}^{o}$ & 79.4660 & 79.4545 & 79.3136 & 79.3994 & 79.3733 & 79.2132 & 79.1927 & 79.1781 \\
\hline 26 & $2 \mathrm{~s}^{2} 2 \mathrm{p}^{5} 3 \mathrm{~d}$ & ${ }^{1} \mathrm{~F}_{3}^{o}$ & 79.5283 & 79.5139 & 79.3771 & 79.4605 & 79.4361 & 79.2746 & 79.2538 & 79.2390 \\
\hline 27 & $2 \mathrm{~s}^{2} 2 \mathrm{p}^{5} 3 \mathrm{~d}$ & ${ }^{1} \mathrm{P}_{1}^{o}$ & 80.0731 & 80.0679 & 79.9972 & 80.0561 & 80.0433 & 79.8589 & 79.8345 & 79.8137 \\
\hline
\end{tabular}

NIST: Experimental energies compiled by the NIST team and available at the website http://www.nist.gov/pml/data/asd.cfm GRASP2K: Calculations of Jonsson et al. [8] with the GRASP2K code

GRASP1: Calculations of Singh and Aggarwal [9] with the GRASP code for 1016 levels

GRASP2: Present calculations with the GRASP code for 3948 levels

FAC1a: Calculations of Singh and Aggarwal [9] with the FAC code for 1112 levels

FAC1b: Present calculations with the FAC code for 1112 levels

FAC2: Present calculations with the FAC code for 17729 levels

FAC3: Present calculations with the FAC code for 93437 levels 
Table B

Comparison of energies (in Ryd) for the lowest 27 levels of Zn XXI.

\begin{tabular}{|c|c|c|c|c|c|c|c|c|c|c|}
\hline Index & Configuration & Level & NIST & GRASP2K & GRASP1 & GRASP2 & FAC1a & FAC1b & FAC2 & FAC3 \\
\hline 1 & $2 s^{2} 2 p^{6}$ & ${ }^{1} \mathrm{~S}_{0}$ & 00.000 & 0.0000 & 0.0000 & 0.0000 & 0.0000 & 0.0000 & 0.0000 & 0.0000 \\
\hline 2 & $2 s^{2} 2 p^{5} 3 s$ & ${ }^{3} \mathrm{P}_{2}^{o}$ & & 77.2445 & 77.0829 & 77.1655 & 77.1622 & 77.0297 & 77.0111 & 76.9965 \\
\hline 3 & $2 s^{2} 2 p^{5} 3 s$ & ${ }^{1} \mathrm{P}_{1}^{o}$ & 77.429 & 77.4220 & 77.2657 & 77.3445 & 77.3546 & 77.2046 & 77.1858 & 77.1717 \\
\hline 4 & $2 s^{2} 2 p^{5} 3 s$ & ${ }^{3} \mathrm{P}_{0}^{0}$ & & 79.0279 & 78.8614 & 78.9477 & 78.9435 & 78.8018 & 78.7802 & 78.7657 \\
\hline 5 & $2 s^{2} 2 p^{5} 3 s$ & ${ }^{3} \mathrm{P}_{1}^{o}$ & 79.131 & 79.1277 & 78.9649 & 79.0489 & 79.0539 & 78.9002 & 78.8784 & 78.8643 \\
\hline 6 & $2 s^{2} 2 p^{5} 3 p$ & ${ }^{3} \mathrm{~S}_{1}$ & & 80.0759 & 79.9196 & 80.0046 & 79.9897 & 79.8713 & 79.8599 & 79.8446 \\
\hline 7 & $2 s^{2} 2 p^{5} 3 p$ & ${ }^{3} \mathrm{D}_{2}$ & & 80.3236 & 80.1804 & 80.2582 & 80.2601 & 80.1232 & 80.1080 & 80.0941 \\
\hline 8 & $2 \mathrm{~s}^{2} 2 \mathrm{p}^{5} 3 \mathrm{p}$ & ${ }^{3} \mathrm{D}_{3}$ & & 80.6297 & 80.4828 & 80.5639 & 80.5583 & 80.4299 & 80.4147 & 80.4004 \\
\hline 9 & $2 s^{2} 2 p^{5} 3 p$ & ${ }^{1} \mathrm{P}_{1}$ & & 80.6955 & 80.5515 & 80.6338 & 80.6322 & 80.4966 & 80.4804 & 80.4663 \\
\hline 10 & $2 s^{2} 2 p^{5} 3 p$ & ${ }^{3} \mathrm{P}_{2}$ & 80.930 & 80.8980 & 80.7564 & 80.8322 & 80.8381 & 80.6971 & 80.6811 & 80.6674 \\
\hline 11 & $2 s^{2} 2 p^{5} 3 p$ & ${ }^{3} \mathrm{P}_{0}$ & & 81.5606 & 81.4461 & 81.5142 & 81.5289 & 81.3700 & 81.3555 & 81.3392 \\
\hline 12 & $2 s^{2} 2 p^{5} 3 p$ & ${ }^{3} \mathrm{D}_{1}$ & & 82.0446 & 81.8956 & 81.9807 & 81.9773 & 81.8353 & 81.8167 & 81.8027 \\
\hline 13 & $2 s^{2} 2 p^{5} 3 p$ & ${ }^{3} \mathrm{P}_{1}$ & & 82.4876 & 82.3366 & 82.4203 & 82.4164 & 82.2758 & 82.2578 & 82.2436 \\
\hline 14 & $2 s^{2} 2 p^{5} 3 p$ & ${ }^{1} \mathrm{D}_{2}$ & 82.568 & 82.5407 & 82.3920 & 82.4740 & 82.4746 & 82.3283 & 82.3098 & 82.2960 \\
\hline 15 & $2 s^{2} 2 p^{5} 3 p$ & ${ }^{1} \mathrm{~S}_{0}$ & 83.453 & 83.4324 & 83.4967 & 83.5049 & 83.5888 & 83.3206 & 83.3191 & 83.2879 \\
\hline 16 & $2 \mathrm{~s}^{2} 2 \mathrm{p}^{5} 3 \mathrm{~d}$ & ${ }^{3} \mathrm{P}_{0}^{o}$ & & 84.3033 & 84.1579 & 84.2407 & 84.2146 & 84.0717 & 84.0589 & 84.0433 \\
\hline 17 & $2 \mathrm{~s}^{2} 2 \mathrm{p}^{5} 3 \mathrm{~d}$ & ${ }^{3} \mathrm{P}_{1}^{0}$ & 84.408 & 84.4092 & 84.2661 & 84.3490 & 84.3240 & 84.1796 & 84.1663 & 84.1506 \\
\hline 18 & $2 \mathrm{~s}^{2} 2 \mathrm{p}^{5} 3 \mathrm{~d}$ & ${ }^{3} \mathrm{~F}_{4}^{o}$ & & 84.5963 & 84.4631 & 84.5385 & 84.5172 & 84.3768 & 84.3630 & 84.3468 \\
\hline 19 & $2 s^{2} 2 p^{5} 3 d$ & ${ }^{3} \mathrm{P}_{2}^{0}$ & & 84.6078 & 84.4671 & 84.5502 & 84.5274 & 84.3802 & 84.3656 & 84.3500 \\
\hline 20 & $2 \mathrm{~s}^{2} 2 \mathrm{p}^{5} 3 \mathrm{~d}$ & ${ }^{3} \mathrm{~F}_{3}^{o}$ & & 84.6385 & 84.5079 & 84.5860 & 84.5635 & 84.4157 & 84.4000 & 84.3840 \\
\hline 21 & $2 \mathrm{~s}^{2} 2 \mathrm{p}^{5} 3 \mathrm{~d}$ & ${ }^{1} \mathrm{D}_{2}^{o}$ & & 84.8102 & 84.6811 & 84.7618 & 84.7391 & 84.5887 & 84.5709 & 84.5552 \\
\hline 22 & $2 \mathrm{~s}^{2} 2 \mathrm{p}^{5} 3 \mathrm{~d}$ & ${ }^{3} \mathrm{D}_{3}^{o}$ & & 84.9321 & 84.8000 & 84.8822 & 84.8624 & 84.7074 & 84.6899 & 84.6745 \\
\hline 23 & $2 \mathrm{~s}^{2} 2 \mathrm{p}^{5} 3 \mathrm{~d}$ & ${ }^{3} \mathrm{D}_{1}^{o}$ & 85.460 & 85.4497 & 85.3457 & 85.4174 & 85.3986 & 85.2347 & 85.2157 & 85.1973 \\
\hline 24 & $2 \mathrm{~s}^{2} 2 \mathrm{p}^{5} 3 \mathrm{~d}$ & ${ }^{3} \mathrm{~F}_{2}^{o}$ & & 86.4039 & 86.2698 & 86.3512 & 86.3332 & 86.1788 & 86.1599 & 86.1440 \\
\hline 25 & $2 \mathrm{~s}^{2} 2 \mathrm{p}^{5} 3 \mathrm{~d}$ & ${ }^{3} \mathrm{D}_{2}^{O}$ & & 86.5016 & 86.3610 & 86.4473 & 86.4209 & 86.2615 & 86.2424 & 86.2270 \\
\hline 26 & $2 \mathrm{~s}^{2} 2 \mathrm{p}^{5} 3 \mathrm{~d}$ & ${ }^{1} \mathrm{~F}_{3}^{o}$ & & 86.5680 & 86.4317 & 86.5155 & 86.4909 & 86.3300 & 86.3106 & 86.2950 \\
\hline 27 & $2 \mathrm{~s}^{2} 2 \mathrm{p}^{5} 3 \mathrm{~d}$ & ${ }^{1} \mathrm{P}_{1}^{o}$ & $87.340^{\mathrm{a}}$ & 87.1303 & 87.0583 & 87.1188 & 87.1054 & 86.9226 & 86.8997 & 86.8781 \\
\hline
\end{tabular}

NIST: Experimental energies compiled by the NIST team and available at the website http://www.nist.gov/pml/data/asd.cfm GRASP2K: Calculations of Jonsson et al. [8] with the GRASP2K code

GRASP1: Calculations of Singh and Aggarwal [9] with the GRASP code for 1016 levels

GRASP2: Present calculations with the GRASP code for 3948 levels

FAC1a: Calculations of Singh and Aggarwal [9] with the FAC code for 1112 levels

FAC1b: Present calculations with the FAC code for 1112 levels

FAC2: Present calculations with the FAC code for 17729 levels

FAC3: Present calculations with the FAC code for 93437 levels

a: see text in Section 2 
Table C

Comparison of energies (in Ryd) for the lowest 27 levels of Ga XXII.

\begin{tabular}{|c|c|c|c|c|c|c|c|c|c|c|}
\hline Index & Configuration & Level & NIST & GRASP2K & GRASP1 & GRASP2 & FAC1a & FAC1b & FAC2 & FAC3 \\
\hline 1 & $2 \mathrm{~s}^{2} 2 \mathrm{p}^{6}$ & ${ }^{1} \mathrm{~S}_{0}$ & 0.000 & 0.0000 & 0.0000 & 0.0000 & 0.0000 & 0.0000 & 0.0000 & 0.0000 \\
\hline 2 & $2 s^{2} 2 p^{5} 3 s$ & ${ }^{3} \mathrm{P}_{2}^{o}$ & & 83.9042 & 83.7426 & 83.8255 & 83.8227 & 83.6908 & 83.6736 & 83.6583 \\
\hline 3 & $2 s^{2} 2 p^{5} 3 s$ & ${ }^{1} \mathrm{P}_{1}^{o}$ & 84.090 & 84.0909 & 83.9348 & 84.0138 & 84.0246 & 83.8748 & 83.8575 & 83.8428 \\
\hline 4 & $2 s^{2} 2 p^{5} 3 s$ & ${ }^{3} \mathrm{P}_{0}^{0}$ & & 85.9727 & 85.8058 & 85.8926 & 85.8890 & 85.7471 & 85.7268 & 85.7116 \\
\hline 5 & $2 s^{2} 2 p^{5} 3 s$ & ${ }^{3} \mathrm{P}_{1}^{0}$ & 86.100 & 86.0756 & 85.9122 & 85.9968 & 86.0023 & 85.8487 & 85.8282 & 85.8134 \\
\hline 6 & $2 s^{2} 2 p^{5} 3 p$ & ${ }^{3} \mathrm{~S}_{1}$ & & 86.8900 & 86.7349 & 86.8197 & 86.8059 & 86.6873 & 86.6773 & 86.6613 \\
\hline 7 & $2 s^{2} 2 p^{5} 3 p$ & ${ }^{3} \mathrm{D}_{2}$ & & 87.1336 & 86.9911 & 87.0691 & 87.0714 & 86.9350 & 86.9213 & 86.9067 \\
\hline 8 & $2 \mathrm{~s}^{2} 2 \mathrm{p}^{5} 3 \mathrm{p}$ & ${ }^{3} \mathrm{D}_{3}$ & & 87.5057 & 87.3592 & 87.4406 & 87.4354 & 87.3076 & 87.2939 & 87.2788 \\
\hline 9 & $2 s^{2} 2 p^{5} 3 p$ & ${ }^{1} \mathrm{P}_{1}$ & & 87.5659 & 87.4221 & 87.5050 & 87.5033 & 87.3686 & 87.3540 & 87.3392 \\
\hline 10 & $2 s^{2} 2 p^{5} 3 p$ & ${ }^{3} \mathrm{P}_{2}$ & 87.786 & 87.7871 & 87.6461 & 87.7222 & 87.7284 & 87.5880 & 87.5735 & 87.5591 \\
\hline 11 & $2 \mathrm{~s}^{2} 2 \mathrm{p}^{5} 3 \mathrm{p}$ & ${ }^{3} \mathrm{P}_{0}$ & & 88.5231 & 88.4146 & 88.4812 & 88.4982 & 88.3369 & 88.3243 & 88.3068 \\
\hline 12 & $2 s^{2} 2 p^{5} 3 p$ & ${ }^{3} \mathrm{D}_{1}$ & & 89.1342 & 88.9853 & 89.0710 & 89.0679 & 88.9259 & 88.9087 & 88.8939 \\
\hline 13 & $2 s^{2} 2 p^{5} 3 p$ & ${ }^{3} \mathrm{P}_{1}$ & & 89.6493 & 89.4964 & 89.5807 & 89.5769 & 89.4364 & 89.4199 & 89.4049 \\
\hline 14 & $2 s^{2} 2 p^{5} 3 p$ & ${ }^{1} \mathrm{D}_{2}$ & 89.725 & 89.7069 & 89.5583 & 89.6410 & 89.6419 & 89.4955 & 89.4783 & 89.4638 \\
\hline 15 & $2 s^{2} 2 p^{5} 3 p$ & ${ }^{1} \mathrm{~S}_{0}$ & & 90.5554 & 90.6161 & 90.6266 & 90.7082 & 90.4443 & 90.4436 & 90.4115 \\
\hline 16 & $2 s^{2} 2 p^{5} 3 d$ & ${ }^{3} \mathrm{P}_{0}^{o}$ & & 91.3482 & 91.2036 & 91.2865 & 91.2602 & 91.1186 & 91.1073 & 91.0909 \\
\hline 17 & $2 s^{2} 2 p^{5} 3 d$ & ${ }^{3} \mathrm{P}_{1}^{0}$ & 91.470 & 91.4642 & 91.3219 & 91.4048 & 91.3798 & 91.2365 & 91.2246 & 91.2082 \\
\hline 18 & $2 \mathrm{~s}^{2} 2 \mathrm{p}^{5} 3 \mathrm{~d}$ & ${ }^{3} \mathrm{~F}_{4}^{-1}$ & & 91.6664 & 91.5338 & 91.6093 & 91.5879 & 91.4488 & 91.4365 & 91.4195 \\
\hline 19 & $2 \mathrm{~s}^{2} 2 \mathrm{p}^{5} 3 \mathrm{~d}$ & ${ }^{3} \mathrm{P}_{2}^{0}$ & & 91.6808 & 91.5411 & 91.6243 & 91.6013 & 91.4552 & 91.4420 & 91.4257 \\
\hline 20 & $2 \mathrm{~s}^{2} 2 \mathrm{p}^{5} 3 \mathrm{~d}$ & ${ }^{3} \mathrm{~F}_{3}^{o}$ & & 91.7010 & 91.5713 & 91.6495 & 91.6270 & 91.4803 & 91.4661 & 91.4493 \\
\hline 21 & $2 \mathrm{~s}^{2} 2 \mathrm{p}^{5} 3 \mathrm{~d}$ & ${ }^{1} \mathrm{D}_{2}^{o}$ & & 91.8856 & 91.7574 & 91.8384 & 91.8157 & 91.6663 & 91.6498 & 91.6334 \\
\hline 22 & $2 \mathrm{~s}^{2} 2 \mathrm{p}^{5} 3 \mathrm{~d}$ & ${ }^{3} \mathrm{D}_{3}^{\circ}$ & & 92.0212 & 91.8898 & 91.9722 & 91.9525 & 91.7983 & 91.7823 & 91.7662 \\
\hline 23 & $2 \mathrm{~s}^{2} 2 \mathrm{p}^{5} 3 \mathrm{~d}$ & ${ }^{3} \mathrm{D}_{1}^{0}$ & 92.600 & 92.5850 & 92.4846 & 92.5555 & 92.5370 & 92.3733 & 92.3557 & 92.3361 \\
\hline 24 & $2 \mathrm{~s}^{2} 2 \mathrm{p}^{5} 3 \mathrm{~d}$ & ${ }^{3} \mathrm{~F}_{2}^{0}$ & & 93.7480 & 93.6143 & 93.6960 & 93.6780 & 93.5243 & 93.5067 & 93.4901 \\
\hline 25 & $2 \mathrm{~s}^{2} 2 \mathrm{p}^{5} 3 \mathrm{~d}$ & ${ }^{3} \mathrm{D}_{2}^{\circ}$ & & 93.8595 & 93.7190 & 93.8057 & 93.7793 & 93.6204 & 93.6027 & 93.5866 \\
\hline 26 & $2 s^{2} 2 p^{5} 3 d$ & ${ }^{1} \mathrm{~F}_{3}^{o}$ & & 93.9328 & 93.7968 & 93.8810 & 93.8564 & 93.6960 & 93.6780 & 93.6617 \\
\hline 27 & $2 \mathrm{~s}^{2} 2 \mathrm{p}^{5} 3 \mathrm{~d}$ & ${ }^{1} \mathrm{P}_{1}^{0}$ & 94.500 & 94.5004 & 94.4270 & 94.4890 & 94.4753 & 94.2939 & 94.2724 & 94.2501 \\
\hline
\end{tabular}

NIST: Experimental energies compiled by the NIST team and available at the website http://www.nist.gov/pml/data/asd.cfm GRASP2K: Calculations of Jonsson et al. [8] with the GRASP2K code

GRASP1: Calculations of Singh and Aggarwal [9] with the GRASP code for 1016 levels

GRASP2: Present calculations with the GRASP code for 3948 levels

FAC1a: Calculations of Singh and Aggarwal [9] with the FAC code for 1112 levels

FAC1b: Present calculations with the FAC code for 1112 levels

FAC2: Present calculations with the FAC code for 17729 levels

FAC3: Present calculations with the FAC code for 93437 levels 
Table D

Comparison of oscillator strengths (f-values) for a few E1 transitions of $\mathrm{Cu}$ XX, Zn XXI and Ga XXII. $a \pm b \equiv a \times 10^{ \pm b}$.

\begin{tabular}{|c|c|c|c|c|c|c|c|}
\hline \multicolumn{2}{|c|}{ Ion } & \multicolumn{2}{|c|}{$\mathrm{Cu} \mathrm{XX}$} & \multicolumn{2}{|c|}{ Zn XXI } & \multicolumn{2}{|c|}{ Ga XXII } \\
\hline I & $\mathrm{J}$ & GRASP & GRASP2K & GRASP & GRASP2K & GRASP & GRASP2K \\
\hline 1 & 3 & $1.31-1$ & $1.28-1$ & $1.32-1$ & $1.29-1$ & $1.33-1$ & $1.30-1$ \\
\hline 1 & 5 & $9.53-2$ & $9.20-2$ & $9.29-2$ & $9.98-2$ & $9.09-2$ & $8.80-2$ \\
\hline 1 & 17 & $9.90-3$ & $1.00-2$ & $9.68-3$ & $9.76-3$ & $9.34-3$ & $9.37-3$ \\
\hline 1 & 23 & $9.03-1$ & $9.24-1$ & $1.00-0$ & $1.02-0$ & $1.10-0$ & $1.11-0$ \\
\hline 1 & 27 & $2.29-0$ & $2.15-0$ & $2.23-0$ & $2.10-0$ & $2.17-0$ & $2.05-0$ \\
\hline 2 & 6 & $4.80-2$ & $4.78-2$ & $4.71-2$ & $4.69-2$ & $4.62-2$ & $4.60-2$ \\
\hline 2 & 7 & $4.58-2$ & $4.52-2$ & $4.44-2$ & $4.38-2$ & $4.30-2$ & $4.25-2$ \\
\hline 2 & 8 & $1.48-1$ & $1.46-1$ & $1.44-1$ & $1.43-1$ & $1.41-1$ & $1.40-1$ \\
\hline 2 & 9 & $1.08-3$ & $9.63-4$ & $5.63-4$ & $4.83-4$ & $2.22-4$ & $1.74-4$ \\
\hline 2 & 10 & $6.23-2$ & $6.13-2$ & $6.05-2$ & $5.98-2$ & $5.90-2$ & $5.81-2$ \\
\hline 2 & 12 & $1.17-4$ & $1.16-4$ & $1.19-4$ & $1.17-4$ & $1.18-4$ & $1.15-4$ \\
\hline 2 & 13 & $3.24-3$ & $3.05-3$ & $2.86-3$ & $2.68-3$ & $2.53-3$ & $2.38-3$ \\
\hline 2 & 14 & $5.61-4$ & $5.35-4$ & $4.65-4$ & $4.44-4$ & $3.90-4$ & $3.72-4$ \\
\hline 3 & 6 & $7.42-4$ & $7.01-4$ & $2.86-4$ & $2.60-4$ & $4.93-5$ & $3.88-5$ \\
\hline 3 & 7 & $8.10-2$ & $8.00-2$ & $7.75-2$ & $7.66-2$ & $7.44-2$ & $7.35-2$ \\
\hline 3 & 9 & $9.97-2$ & $9.85-2$ & $9.76-2$ & $9.65-2$ & $9.56-2$ & $9.46-2$ \\
\hline 3 & 10 & $8.64-2$ & $8.52-2$ & $8.49-2$ & $8.38-2$ & $8.36-2$ & $8.25-2$ \\
\hline 3 & 11 & $3.38-2$ & $3.43-2$ & $3.43-2$ & $3.47-2$ & $3.48-2$ & $3.52-2$ \\
\hline 3 & 12 & $2.85-4$ & $2.40-4$ & $2.46-4$ & $2.09-4$ & $2.15-4$ & $1.83-4$ \\
\hline 3 & 13 & $8.52-5$ & $6.55-5$ & $9.11-5$ & $7.22-5$ & $9.45-5$ & $7.70-5$ \\
\hline 3 & 14 & $6.48-4$ & $6.14-4$ & $5.08-4$ & $4.83-4$ & $4.04-4$ & $3.85-4$ \\
\hline 3 & 15 & $2.12-2$ & $1.87-2$ & $1.94-2$ & $1.71-2$ & $1.77-2$ & $1.55-2$ \\
\hline 4 & 6 & $7.97-4$ & $8.04-4$ & $5.42-4$ & $5.47-4$ & $3.54-4$ & $3.57-4$ \\
\hline 4 & 9 & $9.88-5$ & $9.16-5$ & $4.78-5$ & $4.36-5$ & $2.14-5$ & $1.91-5$ \\
\hline 4 & 12 & $1.04-1$ & $1.01-1$ & $9.80-2$ & $9.58-2$ & $9.29-2$ & $9.10-2$ \\
\hline 4 & 13 & $2.01-1$ & $2.00-1$ & $1.99-1$ & $1.98-1$ & $1.96-1$ & $1.95-1$ \\
\hline 5 & 6 & $3.50-4$ & $3.51-4$ & $2.36-4$ & $2.37-4$ & $1.51-4$ & $1.52-4$ \\
\hline 5 & 7 & $5.07-6$ & $3.67-6$ & $3.52-6$ & $2.56-6$ & $2.45-6$ & $1.79-6$ \\
\hline 5 & 9 & $4.02-5$ & $3.32-5$ & $4.17-5$ & $3.58-5$ & $4.05-5$ & $3.58-5$ \\
\hline 5 & 10 & $5.28-4$ & $5.19-4$ & $3.75-4$ & $3.70-4$ & $2.66-4$ & $2.61-4$ \\
\hline 5 & 11 & $6.82-3$ & $6.04-3$ & $5.83-3$ & $5.11-3$ & $4.89-3$ & $4.26-3$ \\
\hline 5 & 12 & $5.71-2$ & $5.67-2$ & $5.55-2$ & $5.51-2$ & $5.40-2$ & $5.35-2$ \\
\hline 5 & 13 & $3.57-2$ & $3.50-2$ & $3.43-2$ & $3.37-2$ & $3.31-2$ & $3.25-2$ \\
\hline 5 & 14 & $1.78-1$ & $1.76-1$ & $1.74-1$ & $1.72-1$ & $1.71-1$ & $1.69-1$ \\
\hline 5 & 15 & $3.96-2$ & $3.87-2$ & $3.86-2$ & $3.78-2$ & $3.78-2$ & $3.70-2$ \\
\hline
\end{tabular}

GRASP: Present calculations with the GRASP code

GRASP2K: Calculations of Jönsson et al. [8] with the GRASP2K code 
Table E

Comparison of lifetimes $(\tau, \mathrm{s})$ for the lowest 27 levels of Cu XX, Zn XXI and Ga XXII. $a \pm b \equiv a \times 10^{ \pm b}$

\begin{tabular}{|c|c|c|c|c|c|c|c|c|c|c|c|}
\hline \multicolumn{3}{|l|}{ Ion } & \multicolumn{3}{|c|}{$\mathrm{Cu} \mathrm{XX}$} & \multicolumn{3}{|c|}{ Zn XXI } & \multicolumn{3}{|c|}{ Ga XXII } \\
\hline Index & Config. & Level & GRASP $^{a}$ & $\mathrm{GRASP}^{b}$ & $\operatorname{GRASP}^{c}$ & $\operatorname{GRASP}^{a}$ & $\mathrm{GRASP}^{b}$ & $\operatorname{GRASP}^{c}$ & $\operatorname{GRASP}^{a}$ & $\mathrm{GRASP}^{b}$ & GRASP $^{c}$ \\
\hline 1 & $2 \mathrm{p}^{6}$ & ${ }^{1} \mathrm{~S}_{0}$ & & & & & & & & & \\
\hline 2 & $2 \mathrm{p}^{5} 3 \mathrm{~s}$ & ${ }^{3} \mathrm{P}_{2}^{o}$ & $7.896-07$ & $1.588-06$ & $1.47-06$ & $1.089-06$ & $1.133-06$ & $1.05-06$ & $7.896-07$ & $8.203-07$ & 7.64-07 \\
\hline 3 & $2 \mathrm{p}^{5} 3 \mathrm{~s}$ & ${ }^{1} \mathrm{P}_{1}^{o}$ & $5.661-13$ & $5.796-13$ & 5.54-13 & $4.724-13$ & $4.832-13$ & $4.63-13$ & $3.980-13$ & $4.065-13$ & $3.90-13$ \\
\hline 4 & $2 \mathrm{p}^{5} 3 \mathrm{~s}$ & ${ }^{3} \mathrm{P}_{0}^{o}$ & $1.342-05$ & $1.338-05$ & $1.36-05$ & $8.355-06$ & $8.330-06$ & $8.43-06$ & $5.296-06$ & $5.282-06$ & 5.34-06 \\
\hline 5 & $2 \mathrm{p}^{5} 3 \mathrm{~s}$ & ${ }^{3} \mathrm{P}_{1}^{0}$ & $7.477-13$ & $7.728-13$ & $7.25-13$ & $6.435-13$ & $6.642-13$ & $6.25-13$ & $5.557-13$ & $5.729-13$ & $5.41-13$ \\
\hline 6 & $2 p^{5} 3 p$ & ${ }^{3} \mathrm{~S}_{1}^{1}$ & $2.138-10$ & $2.157-10$ & $2.15-10$ & $1.958-10$ & $1.977-10$ & $1.97-10$ & $1.802-10$ & $1.821-10$ & $1.81-10$ \\
\hline 7 & $2 p^{5} 3 p$ & ${ }^{3} \mathrm{D}_{2}$ & $1.197-10$ & $1.367-10$ & $1.34-10$ & $1.211-10$ & $1.235-10$ & $1.21-10$ & $1.093-10$ & $1.113-10$ & $1.09-10$ \\
\hline 8 & $2 p^{5} 3 p$ & ${ }^{3} \mathrm{D}_{3}$ & $1.159-10$ & $1.182-10$ & $1.16-10$ & $1.046-10$ & $1.066-10$ & $1.05-10$ & $9.440-11$ & $9.619-11$ & $9.45-11$ \\
\hline 9 & $2 p^{5} 3 p$ & ${ }^{1} \mathrm{P}_{1}$ & $1.277-10$ & $1.308-10$ & $1.29-10$ & $1.166-10$ & $1.193-10$ & $1.17-10$ & $1.063-10$ & $1.087-10$ & $1.07-10$ \\
\hline 10 & $2 p^{5} 3 p$ & ${ }^{3} \mathrm{P}_{2}$ & $7.921-11$ & $8.700-11$ & $8.51-11$ & $7.555-11$ & $7.708-11$ & 7.55-11 & $6.693-11$ & $6.823-11$ & $6.69-11$ \\
\hline 11 & $2 \mathrm{p}^{5} 3 \mathrm{p}$ & ${ }^{3} \mathrm{P}_{0}$ & $7.538-11$ & 7.614-11 & 7.58-11 & $6.570-11$ & $6.637-11$ & 6.61-11 & $5.720-11$ & $5.782-11$ & $5.75-11$ \\
\hline 12 & $2 p^{5} 3 p$ & ${ }^{3} \mathrm{D}_{1}$ & $1.678-10$ & $1.720-10$ & $1.69-10$ & $1.581-10$ & $1.620-10$ & $1.59-10$ & $1.493-10$ & $1.529-10$ & $1.50-10$ \\
\hline 13 & $2 p^{5} 3 p$ & ${ }^{3} \mathrm{P}_{1}$ & $1.036-10$ & $1.061-10$ & $1.04-10$ & $9.409-11$ & $9.625-11$ & $9.42-11$ & $8.541-11$ & $8.731-11$ & $8.55-11$ \\
\hline 14 & $2 p^{5} 3 p$ & ${ }^{1} \mathrm{D}_{2}$ & 8.613-11 & $9.614-11$ & $9.43-11$ & $8.319-11$ & $8.474-11$ & $8.31-11$ & 7.329-11 & $7.460-11$ & $7.32-11$ \\
\hline 15 & $2 \mathrm{p}^{5} 3 \mathrm{p}$ & ${ }^{1} \mathrm{~S}_{0}$ & $2.921-11$ & $3.342-11$ & $2.76-11$ & $2.761-11$ & $3.150-11$ & 2.61-11 & $2.623-11$ & $2.985-11$ & $2.48-11$ \\
\hline 16 & $2 \mathrm{p}^{5} 3 \mathrm{~d}$ & ${ }^{3} \mathrm{P}_{0}^{o}$ & $6.902-11$ & $6.981-11$ & $6.92-11$ & $6.417-11$ & $6.487-11$ & $6.43-11$ & $5.964-11$ & $6.026-11$ & $5.97-11$ \\
\hline 17 & $2 \mathrm{p}^{5} 3 \mathrm{~d}$ & ${ }^{3} \mathrm{P}_{1}^{0}$ & $5.750-12$ & $5.693-12$ & 5.86-12 & $4.998-12$ & $4.963-12$ & $5.09-12$ & $4.432-12$ & $4.414-12$ & $4.50-12$ \\
\hline 18 & $2 \mathrm{p}^{5} 3 \mathrm{~d}$ & ${ }^{3} \mathrm{~F}_{4}^{o}$ & $7.571-11$ & $7.696-11$ & $7.17-11$ & $7.173-11$ & $7.286-11$ & $6.69-11$ & $6.805-11$ & $6.906-11$ & $6.23-11$ \\
\hline 19 & $2 \mathrm{p}^{5} 3 \mathrm{~d}$ & ${ }^{3} \mathrm{P}_{0}^{0}$ & 7.123-11 & $7.257-11$ & $7.57-11$ & $6.652-11$ & $6.765-11$ & $7.17-11$ & $6.198-11$ & $6.299-11$ & $6.80-11$ \\
\hline 20 & $2 p^{5} 3 d$ & ${ }^{3} \mathrm{~F}_{3}^{o}$ & $6.198-11$ & $6.316-11$ & $6.23-11$ & $5.750-11$ & $5.854-11$ & $5.77-11$ & $5.335-11$ & $5.427-11$ & $5.36-11$ \\
\hline 21 & $2 \mathrm{p}^{5} 3 \mathrm{~d}$ & ${ }^{1} \mathrm{D}_{2}^{o}$ & $6.003-11$ & $8.129-11$ & 6.04-11 & $5.613-11$ & $5.723-11$ & 5.64-11 & $5.257-11$ & $5.356-11$ & $5.28-11$ \\
\hline 22 & $2 \mathrm{p}^{5} 3 \mathrm{~d}$ & ${ }^{3} \mathrm{D}_{3}^{o}$ & $6.693-11$ & $6.842-11$ & 6.76-11 & $6.330-11$ & $6.465-11$ & 6.39-11 & $5.997-11$ & $6.118-11$ & $6.05-11$ \\
\hline 23 & $2 \mathrm{p}^{5} 3 \mathrm{~d}$ & ${ }^{3} \mathrm{D}_{1}^{\circ}$ & $6.693-14$ & $6.535-14$ & $6.85-14$ & $5.099-14$ & $5.010-14$ & $5.19-14$ & $3.958-14$ & $3.910-14$ & $4.01-14$ \\
\hline 24 & $2 \mathrm{p}^{5} 3 \mathrm{~d}$ & ${ }^{3} \mathrm{~F}_{0}^{o}$ & $6.036-11$ & $6.157-11$ & $6.06-11$ & $5.608-11$ & $5.713-11$ & $5.62-11$ & $5.208-11$ & $5.302-11$ & $5.22-11$ \\
\hline 25 & $2 \mathrm{p}^{5} 3 \mathrm{~d}$ & ${ }^{3} \mathrm{D}_{2}^{o}$ & $6.906-11$ & $7.062-11$ & 6.96-11 & $6.555-11$ & $6.694-11$ & $6.60-11$ & $6.226-11$ & $6.352-11$ & $6.26-11$ \\
\hline 26 & $2 \mathrm{p}^{5} 3 \mathrm{~d}$ & ${ }^{1} \mathrm{~F}_{3}^{o}$ & $7.068-11$ & $7.217-11$ & $7.12-11$ & $6.696-11$ & $6.830-11$ & $6.74-11$ & $6.352-11$ & $6.473-11$ & 6.39-11 \\
\hline 27 & $2 \mathrm{p}^{5} 3 \mathrm{~d}$ & ${ }^{1} \mathrm{P}_{1}^{3}$ & $2.548-14$ & $2.708-14$ & $2.48-14$ & $2.208-14$ & $2.342-14$ & $2.15-14$ & $1.929-14$ & $2.041-14$ & $1.88-14$ \\
\hline
\end{tabular}

GRASP $^{a}$ : Present calculations with the GRASP code

GRASP $^{b}$ : Calculations of Jonsson et al. [8] with the GRASP2K code

GRASP $^{c}$ : Calculations of Singh and Aggarwal [9] with the GRASP code 


\section{Explanation of Tables}

Table 1. Energies (Ryd) for the lowest 139 levels of $\mathrm{Cu}$ XX and their lifetimes $(\tau, \mathrm{s})$.

$\begin{array}{ll}\text { Index } & \text { Level Index } \\ \text { Configuration } & \text { The configuration to which the level belongs } \\ \text { Level } & \text { The } L S J \text { designation of the level } \\ \text { GRASP } & \text { Present energies from the GRASP code with } 64 \text { configurations and } 3948 \text { level calculations } \\ \text { FAC } & \text { Present energies from the FAC code with } 93437 \text { level calculations } \\ \tau(\mathrm{s}) & \text { Lifetime of the level in s with the GRASP code }\end{array}$

Table 2. Energies (Ryd) for the lowest 139 levels of Zn XXI and their lifetimes $(\tau, \mathrm{s})$.

$\begin{array}{ll}\text { Index } & \text { Level Index } \\ \text { Configuration } & \text { The configuration to which the level belongs } \\ \text { Level } & \text { The } L S J \text { designation of the level } \\ \text { GRASP } & \text { Present energies from the GRASP code with } 64 \text { configurations and } 3948 \text { level calculations } \\ \text { FAC } & \text { Present energies from the FAC code with } 93437 \text { level calculations } \\ \tau(\mathrm{s}) & \text { Lifetime of the level in s }\end{array}$

Table 3. Energies (Ryd) for the lowest 139 levels of Ga XXII and their lifetimes $(\tau, \mathrm{s})$.

$\begin{array}{ll}\text { Index } & \text { Level Index } \\ \text { Configuration } & \text { The configuration to which the level belongs } \\ \text { Level } & \text { The } L S J \text { designation of the level } \\ \text { GRASP } & \text { Present energies from the GRASP code with } 64 \text { configurations and } 3948 \text { level calculations } \\ \text { FAC } & \text { Present energies from the FAC code with } 38089 \text { level calculations } \\ \tau(\mathrm{s}) & \text { Lifetime of the level in s with the GRASP code }\end{array}$

Table 4. Transition wavelengths $\left(\lambda_{i j}\right.$ in $\AA$ ), radiative rates $\left(A_{j i}\right.$ in $\left.s^{-1}\right)$, oscillator strengths $\left(f_{i j}\right.$, dimensionless), and line strengths ( $\mathrm{S}$, in atomic units) for electric dipole (E1), and $\mathrm{A}_{j i}$ for electric quadrupole (E2), magnetic dipole (M1) and magnetic quadrupole (M2) transitions of Cu XX. The ratio R(E1) of velocity and length forms of A-values for E1 transitions is listed in the last column.

$i$ and $j$
$\lambda_{i j}$
$\mathrm{~A}_{j i}^{E 1}$
$\mathrm{f}_{i j}^{E 1}$
$\mathrm{~S}^{E 1}$
$\mathrm{~A}_{j i}^{E 2}$
$\mathrm{~A}_{j i}^{M 1}$
$\mathrm{~A}_{j i}^{M 2}$
$\mathrm{R}(\mathrm{E} 1)$
$a \pm b$

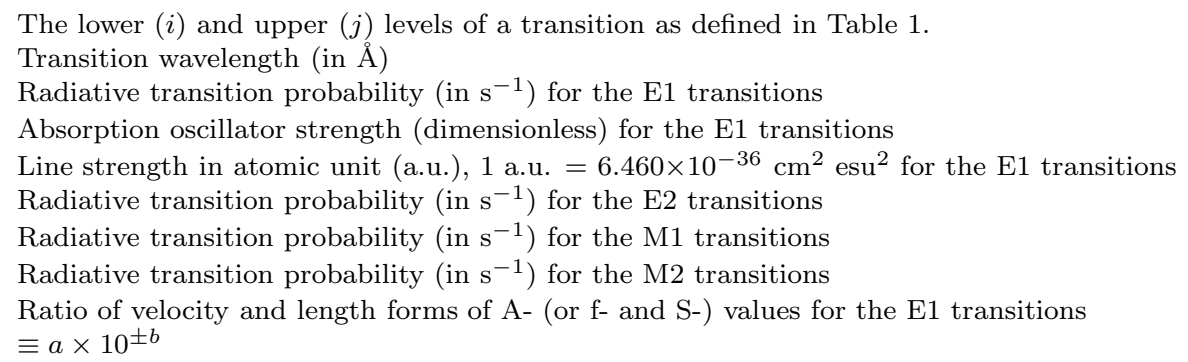


Table 5. Transition wavelengths $\left(\lambda_{i j}\right.$ in $\left.\AA\right)$, radiative rates $\left(\mathrm{A}_{j i}\right.$ in s $\left.{ }^{-1}\right)$, oscillator strengths $\left(\mathrm{f}_{i j}\right.$, dimensionless), and line strengths ( $\mathrm{S}$, in atomic units) for electric dipole (E1), and $\mathrm{A}_{j i}$ for electric quadrupole (E2), magnetic dipole (M1) and magnetic quadrupole (M2) transitions of Zn XXI. The ratio R(E1) of velocity and length forms of A-values for E1 transitions is listed in the last column.

$i$ and $j$
$\lambda_{i j}$
$\mathrm{~A}_{j i}^{E 1}$
$\mathrm{f}_{i j}^{E 1}$
$\mathrm{~S}^{E 1}$
$\mathrm{~A}_{j i}^{E 2}$
$\mathrm{~A}_{j i}^{M 1}$
$\mathrm{~A}_{j i}^{M 2}$
$\mathrm{R}(\mathrm{E} 1)$
$a \pm b$

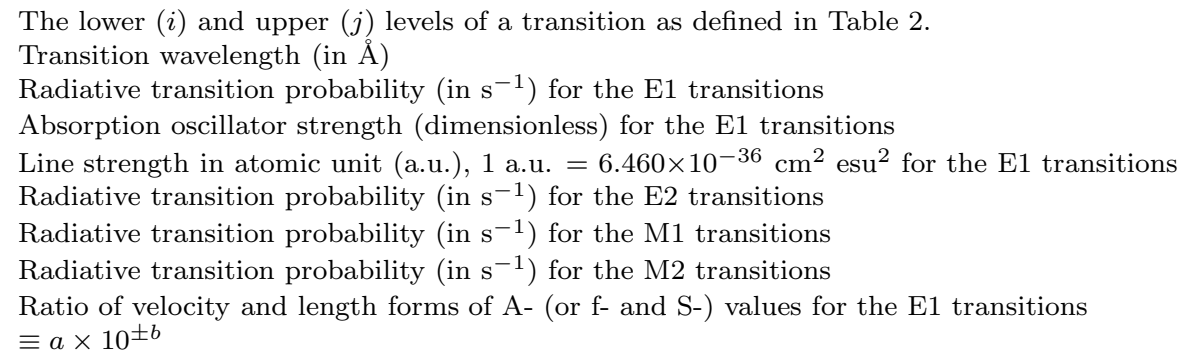

Table 6. Transition wavelengths $\left(\lambda_{i j}\right.$ in $\left.\AA\right)$, radiative rates $\left(\mathrm{A}_{j i}\right.$ in s $\left.{ }^{-1}\right)$, oscillator strengths $\left(\mathrm{f}_{i j}\right.$, dimensionless), and line strengths ( $\mathrm{S}$, in atomic units) for electric dipole (E1), and $\mathrm{A}_{j i}$ for electric quadrupole (E2), magnetic dipole (M1) and magnetic quadrupole (M2) transitions of Ga XXII. The ratio R(E1) of velocity and length forms of A-values for E1 transitions is listed in the last column. 